Please do not remove this page

RMIT

UNIVERSITY

\title{
Examining the use of B-splines in parking assist systems
}

Elbanhawi, Mohamed; Simic, Milan

https://researchrepository.rmit.edu.au/esploro/outputs/9921859480001341/filesAndLinks?institution=61RMIT_INST\&index=null

Elbanhawi, M., \& Simic, M. (2014). Examining the use of B-splines in parking assist systems. Applied Mechanics and Materials, 490-491, 1025-1029.

https://doi.org/10.4028/www.scientific.net/AMM.490-491.1025

Document Version: Accepted Manuscript

Published Version: https://doi.org/10.4028/www.scientific.net/AMM.490-491.1025

Repository homepage: https://researchrepository.rmit.edu.au

(C) 2014 Trans Tech Publications, Switzerland

Downloaded On 2023/04/26 18:31:32 +1000

Please do not remove this page 
Thank you for downloading this document from the RMIT Research Repository.

The RMIT Research Repository is an open access database showcasing the research outputs of RMIT University researchers.

RMIT Research Repository: http://researchbank.rmit.edu.au/

\section{Citation:}

Elbanhawi, M and Simic, M 2014, 'Examining the use of B-splines in parking assist systems', Applied Mechanics and Materials, vol. 490491, pp. 1025-1029.

See this record in the RMIT Research Repository at:

https://researchbank.rmit.edu.au/view/rmit:24157

Version: Accepted Manuscript

Copyright Statement: (c) (2014) Trans Tech Publications, Switzerland

Link to Published Version:

http://dx.doi.org/10.4028/www.scientific.net/AMM.490-491.1025 


\title{
Examining the use of B-splines in Parking Assist Systems
}

\author{
Mohamed Elbanhawi ${ }^{1, a}$ and Milan Simic ${ }^{1, b}$ \\ ${ }^{1}$ School of Aerospace, Mechanical and Manufacturing Engineering, RMIT University, VIC 3083, \\ Australia \\ as3322588@student.rmit.edu.au, ${ }^{\mathrm{b}}$ milan@rmit.edu.au
}

Keywords: Parking Assist, B-splines, Curvature, Intelligent Vehicles

\begin{abstract}
The main objective of the presented study and simulations conducted was to investigate the prospect of using B-spline curves for the automatic parking, i.e. self-driving, or intelligent vehicles. We consider the problem of parallel parking for a non-holonomic vehicle with a known maximum path curvature. The relationship between the properties of the path and the geometry of corresponding parking spot is revealed. The unique properties of B-splines are exploited to synthesize a path that is smooth and of continuous curvature. The contributions of this project are in the generations of better, smooth continuous paths. This improves passenger comfort during the parallel parking maneuver and allow vehicles to park in tighter spots by increasing the feasible range of the parking manoeuver.
\end{abstract}

\section{Introduction}

Parallel parking is considered as one of the most challenging maneuvers that often faces inexperienced and even expert drivers. Improper handling of the vehicle can lead to collision, or the inability to position it correctly in the desired spot. Significant research has made Parking Assist Systems (PAS) accessible due to their obvious commercial, scientific and safety benefits. Automotive companies such as BMW, Volkswagen and Nissan have already introduced PAS in some of their vehicles. These systems can be mainly categorized into semi-automatic and automatic systems. Semi-automatic systems are concerned with generating a path, which is translated into steering wheel control, while the driver controls the accelerator. Automatic systems proceed to generate a trajectory that handles both speed and steering controls, eliminating the need for any driver intervention. These systems are not limited to parking scenarios and can be applied in driverless urban cars. To facilitate autonomous parking lot traversing, researchers have defined systems that generate lane graphs in parking lots $[1,2]$.

The problem of parallel parking has several constraints, such as the collision of the vehicle with any of the obstacles surrounding it, in addition to its kinematic restrictions. The main limitation is that the path's curvature is upper bound, thus smooth paths must be generated to ensure that it is not exceeded. As a result, paths generated for this problem are often discontinuous, which leads to the vehicle stopping during parking. Continuity and smoothness contribute to passenger comfort, which is especially important in cars.

Paths consisting of interconnected segments of arcs, circles and clothoids were used for parking [3]. Even though the paths generated were smooth and continuous, the use of Clothoids is a disadvantage as their synthesis depends on evaluating Fresnel integrals. Clothoids are often evaluated using high order polynomials, or approximated using other types of curves, which increases their computational time and has an adverse effect on their properties [4-7]. Polynomials were proposed, however, fifth order polynomials were found to be the lowest order needed to satisfactory model car's motion [8]. This increases the computational cost of real time curve generation. Polynomials often exhibit oscillations when interpolating a path with close control points [1]. Gómez-Bravo, et al. [9] proposed the use of $\beta$-splines (or Beta Splines). This approach ensured smoothness and computational efficiency, however, it lead to having a limited area where feasible paths can be generated for a given vehicle's curvature, as a result of the use of multiple curve segments to generate a curve similar to circular paths. 
The unique properties of B-splines are the incentive behind their use in various applications. Surprisingly, they are not widely used in robotics, unlike Bezier curves [10-13] and polynomials [1, 14-16]. In our research we have considered their use in parking assist applications. B-spline curves are $C^{2}$ continuous, i.e. they have continuity up to their second derivative. We discuss the process of generating a B-spline curve for parking, while upholding the car's curvature upper limit. The main advantage of this approach is the smoothness and continuity of the curves which allows the vehicle to park in more restricted areas. Methods are also introduced to ensure the path's smoothness which is directly related to the passenger comfort.

\section{Problem Formulation}

Parking Problem. The vehicle, shown in Fig. 1, is required to reverse into a gap between two obstacles. The size of the gap, or parking spot, is assumed to be rectangular of length $L$ and width $W$. The size of the gap relates to the feasibility of the parking maneuver. Additionally the vehicle's curvature is upper bounded, it can be related to its turning radius, see [17] for details. The minimum turning radius, Rmin, of the vehicle, is represented as a dotted circle below, which is another constraint for the parking problem. Finally, the path must ensure that the vehicle will not collide with any obstacles surrounding it, while reversing.

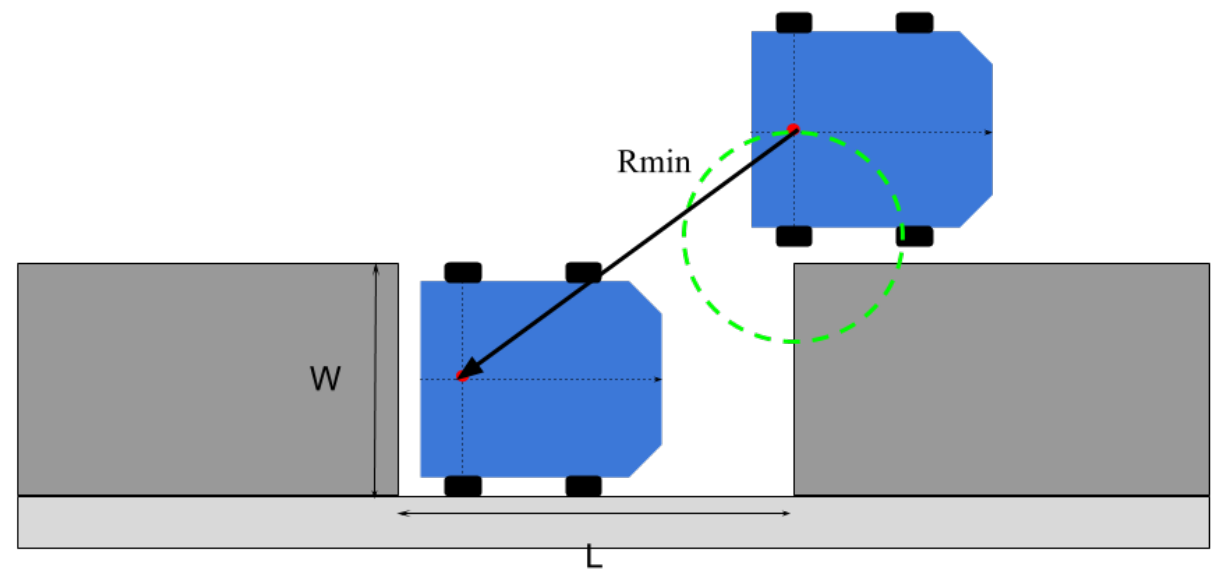

Fig. 1 Parallel Parking

B-Spline Theory. B-splines are piecewise vector valued parametric curves. In order to generate a B-spline curve several points must be defined. They are often referred to as control points. These curves are characterized by their continuity, ability to be modified locally, affine invariance and that they lie within the convex hull of their control polygon A single B-spline curve can be used with any number of control points, as they have no effect on its curvature; see [18] for further details on B-splines properties and synthesis.

A p-th degree B-spline curve, $c(u)$, that is defined by $n$ control points and $n+p+1$ knots $\hat{u}$, is defined as follows, where $u$ is the curve parameter sampled at equal intervals between 0 and 1 .

$$
c(u)=\sum_{i=0}^{n} N_{n, i}(u) P_{i}
$$

$N_{n, i}(u)$ is the B-spline basis function which is defined using the Cox-de Boor algorithm [19].

$$
\begin{gathered}
N_{i, 0}(u)= \begin{cases}1 & u \in\left[\hat{u}_{i}, \hat{u}_{i+1}\right) \\
0 & \text { else }\end{cases} \\
N_{i, p}(u)=\frac{u-u_{i}}{u_{i+p}-u_{i}} N_{i, p-1}(u)+\frac{u_{i+p+1}-u}{u_{i+p+1}-u_{i+1}} N_{i+1, p-1}(u)
\end{gathered}
$$




\section{Proposed Work and Results}

Parking Maneuver. In order to generate a B-spline curve to represent the vehicle's reversing motion into the gap, control points are predefined. The proposal involves a control polyline made up of four points, shown as circle dots in Fig. 2. The control polyline is generated from the right rear wheel. It can be transformed to any point on the vehicle as a result of the affine invariance of B-spline curves. The number of control points is limited to four points, $\boldsymbol{n}=4$, to ensure the path smoothness. As the number of control points increase so will the number of basic functions, as presented in equation 1, which, in turn, exert local control on the path to modify its shape.

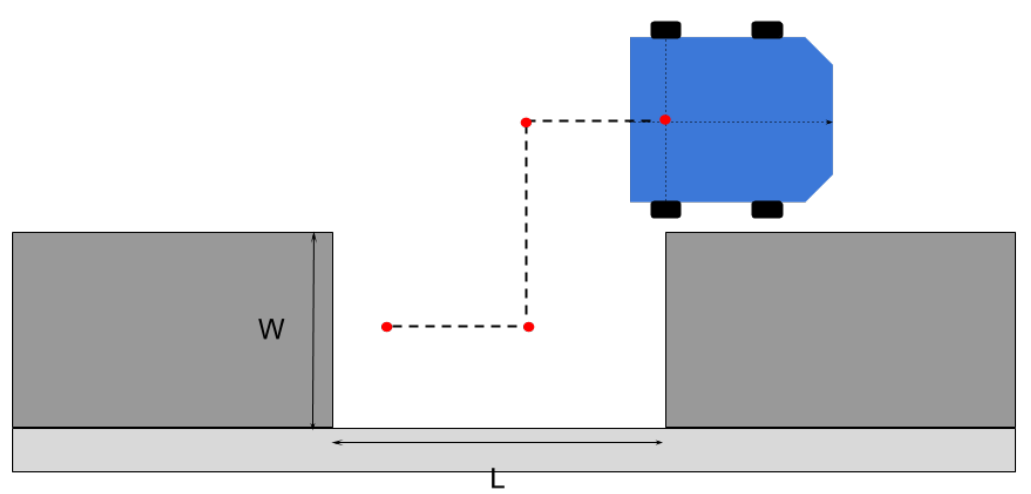

Fig. 2 Parking maneuver primitive control polyline

Second and third degree B-splines can be generated in the case of having four control points. A cubic B-spline is chosen to improve the path's smoothness. The B-spline curve is clamped to pass through the first and last points of the control polyline by having four knot multiplicity at both ends of the knot vector. The resulting B-spline curve and the path's curvature are shown in Fig. 3. The continuous curvature of the path must be highlighted as it improves passenger comfort and prevents the vehicle from stopping. The curve passes through the first and last points and is tangent to the initial and final lines so the vehicle is parallel to the curb before and after parallel parking.
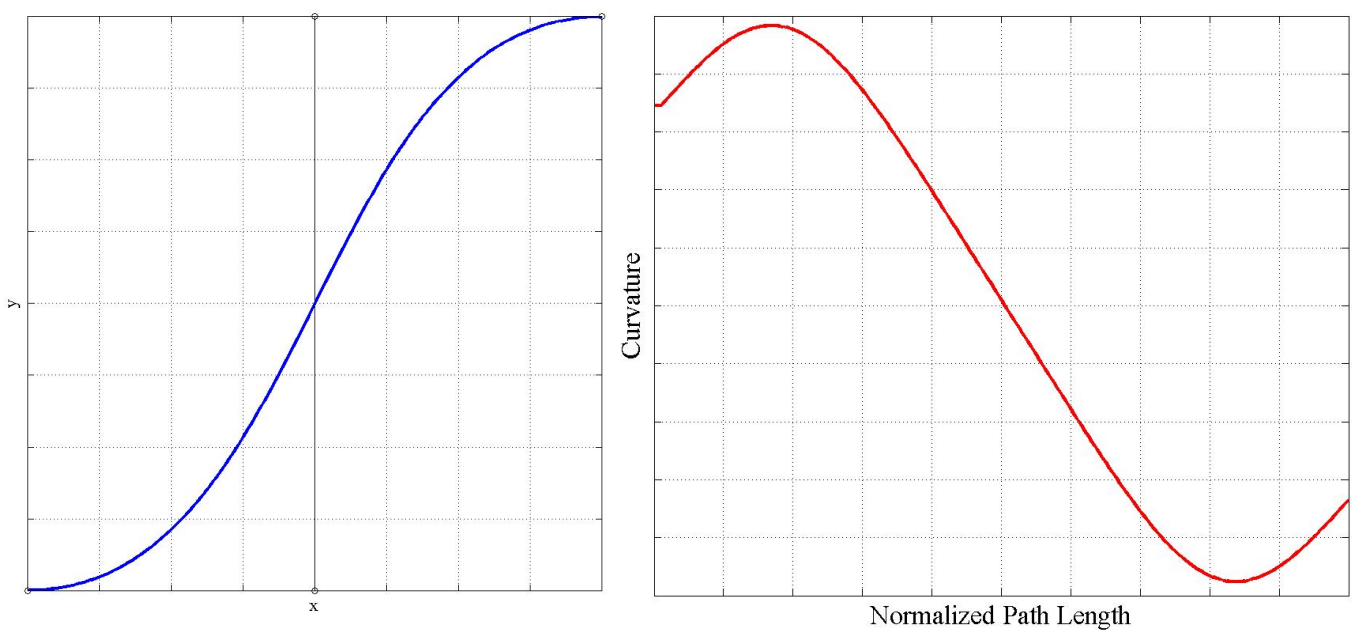

Fig. 3 Clamped cubic B-spline curve on the left and path curvature on the right, representing the parking path

Curvature Control. The curvature of the vehicle is upper-bound, as previously mentioned. The generated path, based on the available parking space, must be studied in relation to that area in order to determine feasible parking spots and their effect on path smoothness. The control points are defined in terms of parking spot parameters $L$ and $W$ which are then varied to study their effect on the generated path smoothness. The results of this experiment were used to define feasible regions for parking and develop methods to improve path smoothness. For this trial a vehicle with maximum curvature of $0.25 \mathrm{~m}^{-1}$ is assumed. Feasible parking spot size, where path does not exceed its maximum curvature, will be defined. From Fig. 2, can be inferred that the minimum parking spot length is equal to the sum of the lengths of the vehicle, $l$, and the distance between the front 
and rear wheels, referred to as the wheel base, $\boldsymbol{w} \boldsymbol{b}$. This is determined by translating the control polyline to the edge of the vehicle and measuring the calculating the required distances.

The effect of changing parking spot parameters, on the corresponding clamped cubic Bspline path curvature, is shown in Fig. 4. It can be noticed that, as the length of the path increases the maximum curvature decreases, unlike the width dependency, which has the opposite effect. The region highlighted in blue corresponds to curvature below the upper limit $\left(0.25 \mathrm{~m}^{-1}\right)$. It is required to find a gap with appropriate dimensions to generate a smooth path. These results can be stored in a look up table and compared with any presented gap to determine parking feasibility. Once a gap is deemed feasible a B-spline curve can be synthesized based on the designed maneuver.
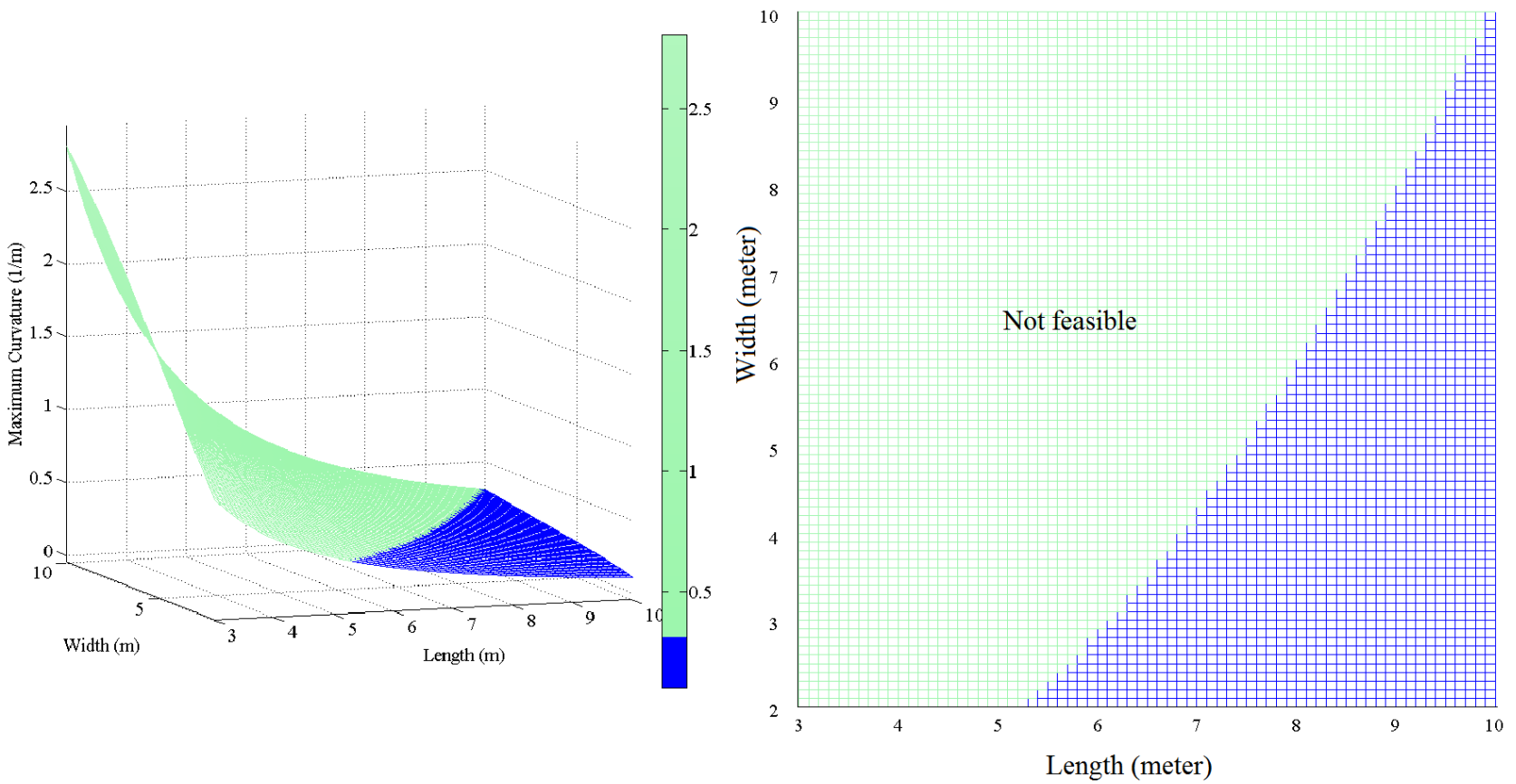

Fig. 4 Effect of changing width and length of parking space on the corresponding B-spline path's curvature

The feasible region, with the proposed solution, is larger than that of the $\beta$-spline based method presented in [9], even when considering only lateral collision. This is a result of using less control points for the parking primitive. A larger feasible region allows for the vehicle to park in tighter parking spots while maintaining its curvature upper limit and following a curvature continuous path.

\section{Conclusions and Future Work}

In this paper we have presented a study on the use of B-spline for developing a PAS for an autonomous self-driving vehicle. A parking primitive is proposed which is a four point control polyline. Appropriate cubic B-spline curve was selected to ensure the smoothness of the path. Bsplines are curve-continuous, which eliminates the need for stopping during parking and improves the comfort of the passenger. Simulation was conducted, to study the effect of the parking spot on the path's curvature, limited for non-holonomic vehicles. The results are promising, showing that smooth, continuous paths can be generated in tight parking spots.

Further investigations could be carried out based on the presented outcomes. Experimental validation of the results will be performed using several robotic research platforms with different kinematic properties. Studying the effect of the use of different curves and approaches on passenger comfort, should be conducted as these vehicles are intended for commercial use. Development of improved systems, that enable vehicle to autonomously navigate a parking lot and proceed to search for a free space, is one of our next applications. It is especially promising with the advancements in graph lane construction, localization algorithms and high resolution sensors technology. 


\section{References}

[1] D. Dolgov, S. Thrun, M. Montemerlo, and J. Diebel, "Path Planning for Autonomous Vehicles in Unknown Semi-structured Environments," The International Journal of Robotics Research, vol. 29, pp. 485-501, April 1, 20102010.

[2] S. Young-Woo, C. Urmson, D. Wettergreen, and L. Jin-Woo, "Building lane-graphs for autonomous parking," in Intelligent Robots and Systems (IROS), 2010 IEEE/RSJ International Conference on, 2010, pp. 6052-6057.

[3] E. Szádeczky-Kardoss and B. Kiss, "Path Planning and Tracking Control for an Automatic Parking Assist System," in European Robotics Symposium 2008. vol. 44, H. Bruyninckx, L. Přeučil, and M. Kulich, Eds., ed: Springer Berlin Heidelberg, 2008, pp. 175-184.

[4] D. S. Meek and D. J. Walton, "An arc spline approximation to a clothoid," Journal of Computational and Applied Mathematics, vol. 170, pp. 59-77, 9/1/ 2004.

[5] L. Z. Wang, K. T. Miura, E. Nakamae, T. Yamamoto, and T. J. Wang, "An approximation approach of the clothoid curve defined in the interval $[0, \pi / 2]$ and its offset by free-form curves," Computer-Aided Design, vol. 33, pp. 1049-1058, 12// 2001.

[6] D. J. Walton and D. S. Meek, "A controlled clothoid spline," Computers \& Graphics, vol. 29, pp. 353-363, 6// 2005.

[7] J. McCrae and K. Singh, "Sketching piecewise clothoid curves," Computers \& Graphics, vol. 33, pp. 452-461, 8// 2009.

[8] Z. Shuwen, M. Simkani, and M. H. Zadeh, "Automatic Vehicle Parallel Parking Design Using Fifth Degree Polynomial Path Planning," in Vehicular Technology Conference (VTC Fall), 2011 IEEE, 2011, pp. 1-4.

[9] F. Gómez-Bravo, F. Cuesta, A. Ollero, and A. Viguria, "Continuous curvature path generation based on -spline curves for parking manoeuvres," Robotics and Autonomous Systems, vol. 56, pp. 360-372, 4/30/ 2008.

[10]Y. Kwangjin and S. Sukkarieh, "An Analytical Continuous-Curvature Path-Smoothing Algorithm," Robotics, IEEE Transactions on, vol. 26, pp. 561-568, 2010.

[11] K. G. Jolly, R. Sreerama Kumar, and R. Vijayakumar, "A Bezier curve based path planning in a multi-agent robot soccer system without violating the acceleration limits," Robotics and Autonomous Systems, vol. 57, pp. 23-33, 1/31/ 2009.

[12]K. Yang, D. Jung, and S. Sukkarieh, "Continuous curvature path-smoothing algorithm using cubic B zier spiral curves for non-holonomic robots," Advanced Robotics, vol. 27, pp. 247258, 2013/03/01 2013.

[13]M. Lepetič, G. Klančar, I. Škrjanc, D. Matko, and B. Potočnik, "Time optimal path planning considering acceleration limits," Robotics and Autonomous Systems, vol. 45, pp. 199-210, 12/31/ 2003.

[14]E. Papadopoulos, I. Poulakakis, and I. Papadimitriou, "On Path Planning and Obstacle Avoidance for Nonholonomic Platforms with Manipulators: A Polynomial Approach," The International Journal of Robotics Research, vol. 21, pp. 367-383, April 1, 20022002.

[15] M. Montemerlo, J. Becker, S. Bhat, H. Dahlkamp, D. Dolgov, S. Ettinger, et al., "Junior: The Stanford Entry in the Urban Challenge," in The DARPA Urban Challenge. vol. 56, M. Buehler, K. Iagnemma, and S. Singh, Eds., ed: Springer Berlin Heidelberg, 2009, pp. 91123.

[16]C. Urmson, J. Anhalt, D. Bagnell, C. Baker, R. Bittner, M. N. Clark, et al., "Autonomous Driving in Urban Environments: Boss and the Urban Challenge," in The DARPA Urban Challenge. vol. 56, M. Buehler, K. Iagnemma, and S. Singh, Eds., ed: Springer Berlin Heidelberg, 2009, pp. 1-59.

[17]R. N. Jazar, Vehicle Dynamics: Theory and Application: Springer, 2008.

[18] G. Farin, Curves and Surfaces for CAGD: Morgan Kaufmann, 2002.

[19] C. De Boor, "On calculating with B-splines," Journal of Approximation Theory, vol. 6, pp. 5062, // 1972. 